Article

\title{
Chronic Sublethal Effects of Cantharidin on the Diamondback Moth Plutella xylostella (Lepidoptera: Plutellidae)
}

\section{Zhengyu Huang and Yalin Zhang *}

Key Laboratory of Plant Protection Resources and Pest Management of the Ministry of Education, College of Plant Protection, Northwest A\&F University, Yangling 712100, Shaanxi, China; E-Mail: huangzy0503@163.com

* Author to whom correspondence should be addressed; E-Mail: yalinzh@nwsuaf.edu.cn; Tel./Fax: +86-29-8709-2190.

Academic Editor: Andreimar M. Soares

Received: 14 April 2015 / Accepted: 26 May 2015 / Published: 29 May 2015

\begin{abstract}
The diamondback moth, Plutella xylostella (Linnaeus) (Lepidoptera: Plutellidae), is a major pest of cruciferous vegetables worldwide. Cantharidin, a natural toxin isolated from blister beetles, has been reported to be toxic to P. xylostella. However, little is known on the chronic sublethal effects of cantharidin on this species. In this study, we assessed the changes of susceptibility, development, reproduction and other demographic parameters in both the selected P. xylostella strain (Sub, selected by $\mathrm{LC}_{25}$ cantharidin for consecutive 12 generations) and the revertant strain (SubR, derived from the Sub strain without being exposed to cantharidin for 12 generations). Results revealed that the two strains maintained a relatively high-level susceptibility to cantharidin. Severe adverse effects on the population dynamics and fitness in Sub strain were observed. In addition, repeated exposure of $P$. xylostella to sublethal concentration of cantharidin resulted in negative effects on adult performance and deformities in adults. Although morphologically normal for individuals, the SubR strain exhibited a disadvantage in population growth rate. Our results showed that sublethal concentration of cantharidin exhibited severe negative effects on population growth for longtime. These findings would be useful for assessing the potential effects and risk of cantharidin on P. xylostella and for developing effective integrated pest management.
\end{abstract}

Keywords: sublethal effects; multigeneration; susceptibility; demographic parameters; Integrated pest management 


\section{Introduction}

The diamondback moth, Plutella xylostella (L.), is regarded as the most destructive pest of cruciferous vegetables throughout the world due to its high rate of consumption, high fecundity, and short generation time (up to 20 generations per year) [1-3]. Currently, its control is not always adequate, probably owing to the development of multiple and cross-resistance to nearly all groups of pesticides applied in the field such as organophosphates, carbamates, pyrethroids, diacylhydrazines, spinosyns and Bacillus thuringiensis, etc. [4].

The natural toxicant cantharidin, an active constituent of blister beetles, has been found to be toxic to some pests [5-8]. Recently, advances in toxicology are changing the way we evaluate toxicants [9]. Besides direct mortality, exposure to a toxicant can result in multiple sublethal effects on population dynamics through impairment to physiological traits, such as: life span [10], development rate [11], fecundity [12,13], and egg hatchability [14]. These effects can provide a comprehensive understanding and prediction of the total effects of a toxicant at the population level $[9,15,16]$.

Recent studies demonstrated that a sublethal concentration of cantharidin significantly affected the development and reproduction of insects including M. separate, H. armigera and P. xylostella [17-19]. However, these studies only covered a period of a few days, leaving us without knowledge of the effects of chronic exposure of this toxicant on insects. The chronic exposure studies mimicked environmentally realistic conditions and monitored the effects of repeated exposure to toxicants over long time periods. Some studies have demonstrated that repeated exposure to sublethal concentrations of toxicants may lead to lower susceptibility [20] or enhanced population growth [21,22]. In addition, the insects can recover their biological characteristics and fitness along with restoring susceptibility when the toxicants are removed [23]. These changes could be major factors in recurrent pest outbreaks and resurgences [22,24-26]. Therefore, sublethal long-term effects analysis of toxicants, estimating the total effect of insecticides on populations, is crucial when choosing new pesticides for controlling pests.

In this study, the stability of susceptibility was investigated in both a cantharidin-selected strain (Sub strain) and a revertant strain (SubR strain). In addition, chronic experiments were conducted to assess serial changes of development, reproduction and other demographic parameters for $P$. xylostella in the presence or absence of cantharidin exposure. We aim to investigate the sublethal effects of cantharidin on P. xylostella over multiple generations and determine how they changed with time in the presence or absence of this stressor.

\section{Results}

\subsection{Susceptibility of SS, Sub and SubR Strains to Cantharidin}

The $48 \mathrm{~h} \mathrm{LC} 25$ of cantharidin for the cantharidin-susceptible strain (SS strain) was $3.84 \mathrm{mg} \cdot \mathrm{L}^{-1}$, and this concentration was chosen for a selection experiment (Table 1). After being selected for 12 generations, the $\mathrm{LC}_{50}$ value of the Sub-12 was higher than that of the SS, but their $95 \%$ confidence interval $(\mathrm{CI})$ values overlapped, suggesting that their $\mathrm{LC}_{50}$ values were not significantly different $(p<0.05)$. The slope of the regression curve increased $31.76 \%$, indicating that the individuals of the sub strain increased in homogeneity when selection cycles were increased. In the first two revertant generations, the $\mathrm{LC}_{50}$ value of cantharidin declined from $18.24 \mathrm{mg} \cdot \mathrm{L}^{-1}$ to about $11.00 \mathrm{mg} \cdot \mathrm{L}^{-1}$ in the 
absence of exposure. The $\mathrm{LC}_{50}$ value was maintained at similar level in the 12 th revertant generation (Table 1).

Table 1. Susceptibility of SS (cantharidin-susceptible strain), Sub (cantharidin-selected strain) and SubR (revertant strain) strains of Plutella xylostella to cantharidin (48 h).

\begin{tabular}{|c|c|c|c|c|c|c|}
\hline Strain & $\mathbf{N}^{\mathrm{a}}$ & Slope \pm SE & $\begin{array}{c}\text { LC }_{50}(95 \% \text { confidence interval }) \\
\left(\mathrm{mg} \cdot \mathrm{L}^{-1}\right) \\
\end{array}$ & $\chi^{2}$ & Toxicity ratio $^{b}$ & $\begin{array}{c}\mathrm{LC}_{25}(95 \% \text { confidence interval }) \\
\left(\mathrm{mg} \cdot \mathrm{L}^{-1}\right)\end{array}$ \\
\hline $\mathrm{SS}^{\mathrm{c}}$ & 320 & $1.48 \pm 0.17$ & $10.95(8.35-15.01)$ & 4.01 & 1.00 & $3.84(2.73-5.07)$ \\
\hline Sub- $6^{d}$ & 320 & $1.50 \pm 0.17$ & $11.34(8.66-15.57)$ & 4.09 & 1.04 & $4.02(2.87-5.29)$ \\
\hline Sub-12 ${ }^{e}$ & 320 & $1.90 \pm 0.20$ & $18.24(14.51-23.64)$ & 5.11 & 1.67 & $8.04(6.08-10.16)$ \\
\hline SubR-1 ${ }^{\mathrm{f}}$ & 320 & $1.59 \pm 0.18$ & $12.11(9.35-16.41)$ & 1.98 & 1.07 & $4.57(3.33-5.95)$ \\
\hline SubR-2 $g$ & 320 & $1.53 \pm 0.17$ & $10.22(7.87-13.77)$ & 3.25 & 1.03 & $3.71(2.65-4.87)$ \\
\hline SubR-6 ${ }^{\mathrm{h}}$ & 320 & $1.63 \pm 0.17$ & $8.89(6.95-11.68)$ & 4.69 & 0.81 & $3.43(2.49-4.45)$ \\
\hline SubR-12 ${ }^{i}$ & 320 & $1.56 \pm 0.17$ & $11.01(9.35-16.41)$ & 3.59 & 1.05 & $4.06(2.93-5.31)$ \\
\hline
\end{tabular}

${ }^{a}$ Number of larvae tested; ${ }^{b} \mathrm{LC}_{50}$ of selected strain/LC $\mathrm{L}_{50}$ of SS strain; ${ }^{\mathrm{c}} \mathrm{SS}$, cantharidin-susceptible strain;

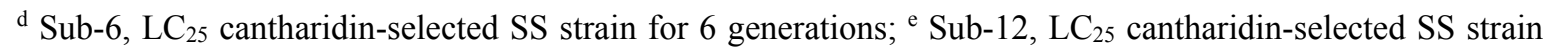
for 12 generations; ${ }^{\mathrm{f}}$ SubR-1, a revertant strain, derived from a substrain of Sub-12 which had not been exposed to cantharidin or any other insecticides for one generation; ${ }^{\mathrm{g}} \mathrm{SubR}-2$, a revertant strain, derived from a substrain of Sub-12 which had not been exposed to cantharidin or any other insecticides for two consecutive generations; ${ }^{\mathrm{h}}$ SubR-6, a revertant strain, derived from a substrain of Sub-12 which had not been exposed to cantharidin or any other insecticides for 6 consecutive generations; ${ }^{i}$ SubR-12, a revertant strain, derived from a substrain of Sub-12 which had not been exposed to cantharidin or any other insecticides for 12 consecutive generations.

\subsection{Effects of Cantharidin on Life History of Different Strains}

The developmental durations of the Sub and SubR strains changed across generations (Table 2). Negative effects were observed in Sub strain. Development of the immature stages was decelerated in both sexes compared to the SS strain. This decelerated development of immature stages primarily appeared in the extension of larval and pupal periods. These effects were significant in the first generation, but gradually faded in latter generations. Additionally, when treated with cantharidin, the adult longevity of females was remarkably reduced in the Sub strain. Cantharidin also significantly influenced the duration of the adult total preoviposition period (TPOP) of P. xylostella in Sub-1 and Sub-6. However, the sublethal effects on Sub-12 were diminished and exhibited no significant difference in comparison to the SS strain, except for the pupal period and adult longevity of females. SubR strain showed a similar duration of whole development in comparison to the SS strain. The developmental durations of eggs were prolonged in both Sub and SubR strains, but the difference from that of the SS strain was not significant (Table 2). 
Table 2. The effects of cantharidin on development (means \pm SE) of Plutella xylostella in different strains.

\begin{tabular}{|c|c|c|c|c|c|c|c|c|c|}
\hline \multirow{3}{*}{ Strain } & \multicolumn{9}{|c|}{ Life stage } \\
\hline & \multirow{2}{*}{ Egg } & \multirow{2}{*}{ Larva } & \multirow{2}{*}{ Pupa } & \multicolumn{2}{|c|}{ Duration of immature stage } & \multicolumn{2}{|c|}{ Adult longevity } & \multirow{2}{*}{$\begin{array}{l}\text { APOP }{ }^{1} \\
\text { Female }\end{array}$} & \multirow{2}{*}{$\begin{array}{l}\text { TPOP }^{2} \\
\text { Female }\end{array}$} \\
\hline & & & & Female & Male & Female & Male & & \\
\hline SS & $3.29 \pm 0.02 \mathrm{a}$ & $7.59 \pm 0.04 \mathrm{c}$ & $4.21 \pm 0.02 \mathrm{c}$ & $15.51 \pm 0.07 \mathrm{bc}$ & $14.80 \pm 0.11 \mathrm{c}$ & $13.34 \pm 0.21 \mathrm{a}$ & $14.01 \pm 0.28 \mathrm{a}$ & $0.59 \pm 0.06 \mathrm{~b}$ & $16.09 \pm 0.12 \mathrm{c}$ \\
\hline Sub-1 & $3.33 \pm 0.05 \mathrm{a}$ & $8.49 \pm 0.08 \mathrm{a}$ & $4.58 \pm 0.04 \mathrm{a}$ & $16.84 \pm 0.13 \mathrm{a}$ & $16.03 \pm 0.12 \mathrm{a}$ & $9.77 \pm 0.36 \mathrm{~d}$ & $11.93 \pm 0.30 \mathrm{~b}$ & $0.93 \pm 0.05 \mathrm{a}$ & $17.77 \pm 0.14 \mathrm{a}$ \\
\hline Sub-6 & $3.45 \pm 0.07 \mathrm{a}$ & $7.97 \pm 0.09 \mathrm{~b}$ & $4.33 \pm 0.06 \mathrm{bc}$ & $15.93 \pm 0.10 b$ & $15.57 \pm 0.09 \mathrm{~b}$ & $10.90 \pm 0.24 \mathrm{~cd}$ & $13.01 \pm 0.53 \mathrm{a}$ & $0.86 \pm 0.06 \mathrm{ab}$ & $16.79 \pm 0.11 \mathrm{~b}$ \\
\hline Sub-12 & $3.39 \pm 0.02 \mathrm{a}$ & $7.67 \pm 0.04 \mathrm{c}$ & $4.42 \pm 0.02 \mathrm{ab}$ & $15.52 \pm 0.10 \mathrm{bc}$ & $15.21 \pm 0.13 \mathrm{bc}$ & $11.90 \pm 0.31 \mathrm{bc}$ & $13.50 \pm 0.19 \mathrm{a}$ & $0.74 \pm 0.09 \mathrm{ab}$ & $16.26 \pm 0.05 \mathrm{c}$ \\
\hline SubR-12 & $3.38 \pm 0.02 \mathrm{a}$ & $7.71 \pm 0.05 \mathrm{bc}$ & $4.25 \pm 0.03 \mathrm{c}$ & $15.31 \pm 0.11 \mathrm{c}$ & $15.30 \pm 0.08 \mathrm{~b}$ & $12.33 \pm 0.27 \mathrm{ab}$ & $13.95 \pm 0.23 \mathrm{a}$ & $0.70 \pm 0.09 \mathrm{ab}$ & $16.00 \pm 0.17 \mathrm{c}$ \\
\hline$p$ & 0.084 & $<0.0001$ & $<0.0001$ & $<0.0001$ & $<0.0001$ & $<0.0001$ & 0.001 & 0.018 & $<0.0001$ \\
\hline$F$ & 2.329 & 33.766 & 14.452 & 34.460 & 18.045 & 11.910 & 6.765 & 3.648 & 35.313 \\
\hline$d f$ & 4,25 & 4,25 & 4,25 & 4,25 & 4,25 & 4,25 & 4,25 & 4,25 & 4,25 \\
\hline
\end{tabular}

Means marked with different letters within the same column are significantly different ( $p<0.05$; Tukey's HSD test). ${ }^{1}$ APOP, adult preoviposition period, time between adult emergence and first oviposition; ${ }^{2}$ TPOP, total preoviposition period, time from birth to first reproduction in female. 


\subsection{Effects of Cantharidin on Adult Performance and Reproductive Potential of Different Strains}

Cantharidin exhibited a significant effect on the reproductive parameters of female adults (Table 3). The oviposition period in both Sub and SubR strains tended to be reduced, even though this reduction gradually became not significant with the increase in selected generations. The reproductive potential investigation mainly included fecundity (total number of eggs laid per female), fertility (the percentage of eggs hatched) and egg size. It is worth noting that the egg size of Sub and SubR strain was obviously different from SS strains (Table 3). The eggs from the Sub strain, especially in the 1st generation, were much smaller. Thereafter, this size recovered slightly in the 6th generation and fluctuated at around $1.23 \times 10^{-2} \mathrm{~mm}^{3}$ in the following generations. The Sub strain accumulated a decrease in fertility accompanying the incremental selection generations. The fertility in Sub-12 was significantly decreased by $11.00 \%$. However, the fertility in SubR-12 recovered but was still significantly lower than in the SS strain. Compared with the SS strain, the reproductive effort per female was significantly lower in Sub-1 and Sub-6. However, there was no difference found among the Sub-12 and SubR-12 cohorts (Table 3).

Table 3. The effects of cantharidin on adult fitness and reproduction potential (means $\pm \mathrm{SE}$ ) of Plutella xylostella in different strains

\begin{tabular}{|c|c|c|c|c|c|}
\hline Strain & Fecundity ${ }^{1}$ & $\begin{array}{c}\text { Egg Size } \\
\left(10^{-2} \mathrm{~mm}^{3}\right)^{2} \\
\end{array}$ & $\begin{array}{c}\text { Reproductive effort } \\
\text { per female }^{3} \\
\end{array}$ & $\begin{array}{c}\text { Oviposition duration } \\
\text { in days } \\
\end{array}$ & $\begin{array}{c}\text { Fertility } \\
\text { (\% egg hatch) }\end{array}$ \\
\hline SS & $203.97 \pm 3.40 \mathrm{ab}$ & $1.32 \pm 0.02 \mathrm{a}$ & $2.69 \pm 0.06 \mathrm{a}$ & $8.37 \pm 0.15 \mathrm{a}$ & $86.86 \pm 0.75 \mathrm{a}$ \\
\hline Sub-1 & $161.26 \pm 2.43 \mathrm{c}$ & $1.21 \pm 0.02 \mathrm{~b}$ & $1.96 \pm 0.05 \mathrm{c}$ & $6.95 \pm 0.17 \mathrm{~b}$ & $85.80 \pm 0.86 \mathrm{ab}$ \\
\hline Sub-6 & $187.41 \pm 5.67 \mathrm{~b}$ & $1.24 \pm 0.02 b$ & $2.33 \pm 0.10 \mathrm{~b}$ & $7.18 \pm 0.17 b$ & $83.46 \pm 1.10 \mathrm{ab}$ \\
\hline Sub-12 & $217.15 \pm 7.87 \mathrm{a}$ & $1.22 \pm 0.02 b$ & $2.64 \pm 0.08 \mathrm{a}$ & $7.79 \pm 0.25 \mathrm{ab}$ & $75.86 \pm 1.44 \mathrm{c}$ \\
\hline SubR-12 & $195.38 \pm 4.99 \mathrm{ab}$ & $1.25 \pm 0.02 \mathrm{~b}$ & $2.43 \pm 0.07 \mathrm{ab}$ & $7.89 \pm 0.16 \mathrm{a}$ & $82.34 \pm 0.52 b$ \\
\hline$p$ & $<0.0001$ & 0.001 & $<0.0001$ & $<0.0001$ & $<0.0001$ \\
\hline$F$ & 18.752 & 6.214 & 15.939 & 9.624 & 19.175 \\
\hline$d f$ & 4,25 & 4,25 & 4,25 & 4,25 & 4,25 \\
\hline
\end{tabular}

Means marked with different letters within the same column are significantly different ( $p<0.05$; Tukey's HSD test);

${ }^{1}$ Total number of eggs laid by each female; ${ }^{2}$ Eggs laid on the first oviposition day; ${ }^{3}$ Fecundity $\times$ egg size.

\subsection{Effects of Cantharidin on Age-Stage Survival Rates $\left(s_{x j}\right)$ of Different Strains}

Lower survival curves in all stages were observed in the Sub strains (Figure 1). It was noticeable that in Sub-1 and Sub-6, the maximum values of survival rate at larval and pupal stages were less than those of the SS.

In addition, negative effects of cantharidin were observed during the adult stage for both sexes. Under sublethal-selected conditions, both females and males exhibited shorter survival periods and lower survival rates. However, these negative effects diminished with the increase in selection generations. Survival rates of the SubR strain at the immature stage were similar to that of the SS strain. 

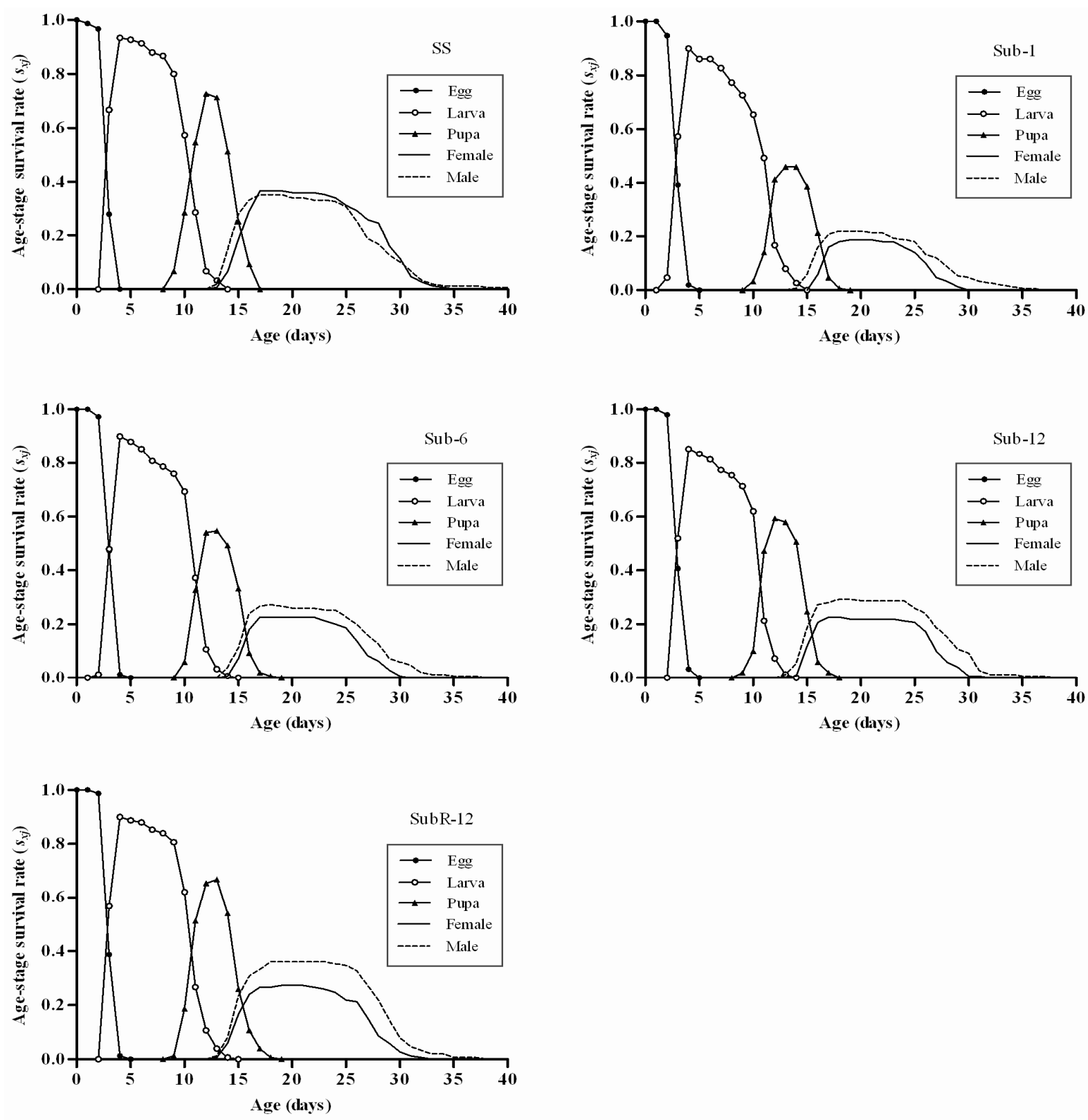

Figure 1. Age-stage survival rates $\left(s_{x j}\right)$ of Plutella xylostella in different strains.

\subsection{Effects of Cantharidin on Age-Specific Survival Rate $\left(l_{x}\right)$, Age-Specific Fecundity $\left(m_{x}\right)$ and} Age-Specific Maternity $\left(l_{x} m_{x}\right)$ of Different Strains

The $l_{x}$ of the Sub strain was also negatively affected by cantharidin (Figure 2). However, the results showed $P$. xylostella survived and reproduced successfully in all strains. The sublethal cantharidin treatment did not interrupt the life cycle of $P$. xylostella. In the SS strain, $l_{x}$ decreased gradually along the whole life course of $P$. xylostella. The trends of $l_{x}$ were similar between the Sub and SS strains. Along with the increase of selection generations, $l_{x}$ was reverted in comparison with that of SS. Mortalities of SubR and SS strains were similar throughout the whole life course. 

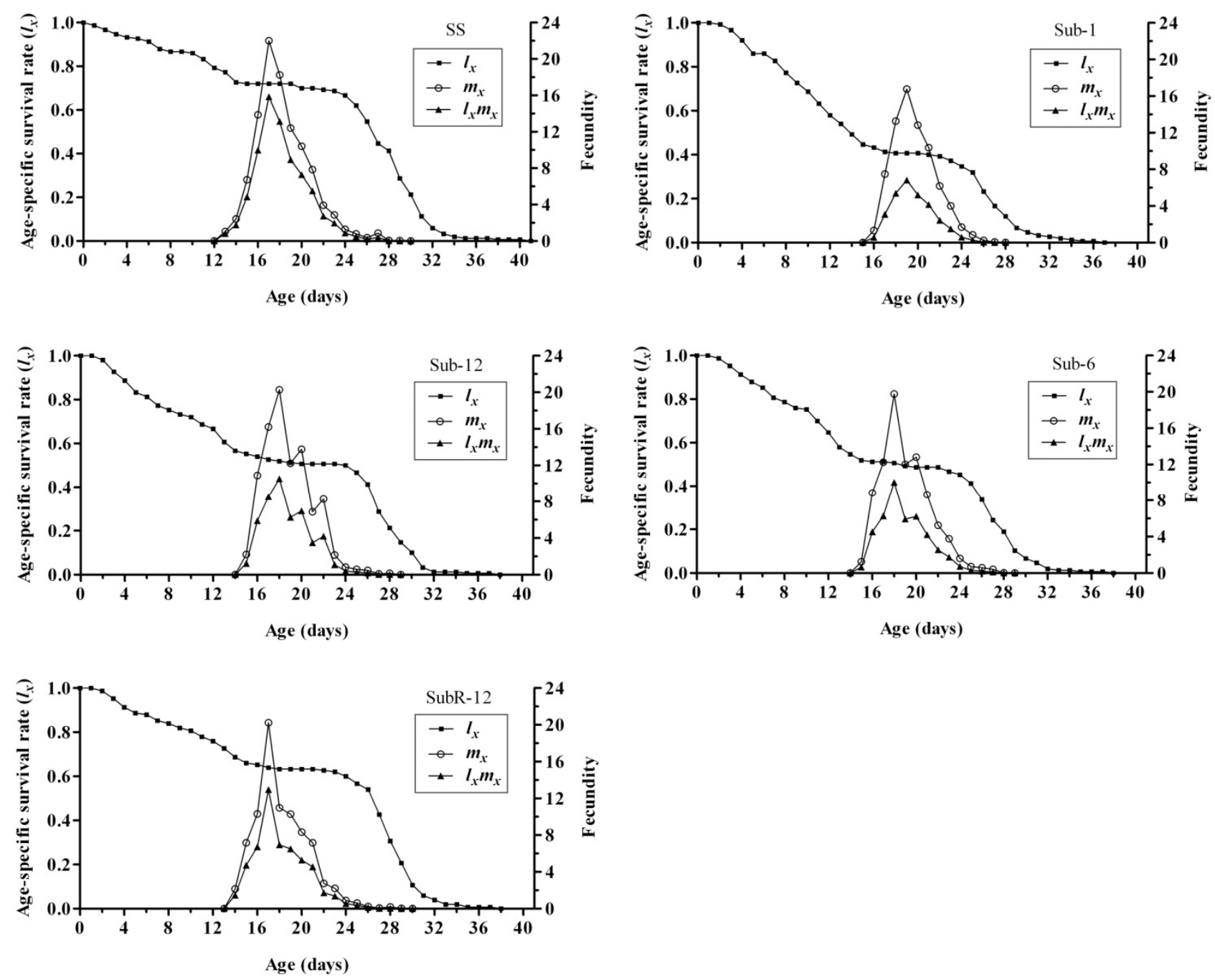

Figure 2. Age-specific survival rate $\left(l_{x}\right)$, age-specific fecundity $\left(m_{x}\right)$ and age-specific maternity $\left(l_{x} m_{x}\right)$ of Plutella xylostella in different strains.

When taking fertility into consideration, the negative effect of cantharidin was evident in age-specific fecundity $\left(m_{x}\right)$ (Figure 2). In SS, females began to oviposit on day 13 and the age-specific fecundity rapidly reached a peak at 22.02 eggs per female on day 17 , followed by a continual fall until death. Sub, SubR and SS strains possessed a similar trend in the age-specific fecundity. However, peak values in the former two strains were lower. The initial time of oviposition in different cohorts (Sub-1, Sub-6, Sub-12, SubR-12), on the 16th, 15th, 15th and 14th day respectively, lagged behind. The cohorts from Sub strain (Sub-1, Sub-6, Sub-12) reached their climax on day 19th, 18th, and 18th, respectively, with peak time being later than that of SS (Figure 2).

\subsection{Effects of Cantharidin on Demographic Parameters and Fitness}

Comparison of various demographic parameters among the SS, Sub and SubR strains revealed significant differences that might be correlated with the population fitness (Table 4). In different cohorts (Sub-1, Sub-6, Sub-12, SubR-12), the demographic parameters of P. xylostella including net reproduction rate $\left(R_{0}\right)$, intrinsic rate of increase $(r)$, finite rate of increase $(\lambda)$ and gross reproduction rate (GRR) tended to be lower than those of the SS. In addition, mean generation time $(T)$ was prolonged. 
Sublethal effects of cantharidin on the Sub strain tended to decline with the increase in selection cycles. Clearly, there were significant differences in all mentioned parameters between the SS and Sub strains except for GRR. In absence of the cantharidin selection, the population characteristics in the SubR strain had recovered to some degree when compared with those of the Sub strain. The rate of relative fitness reflected a recovery in the SubR strain as well (Table 4). However, the SubR strain still exhibited a serious disadvantage in population growth rate compared to the SS strain including decreased $R_{0}, r$ and $\lambda$.

Table 4. Sublethal effects of cantharidin on the demographic parameters (means $\pm \mathrm{SE}$ ) in different Plutella xylostella strains

\begin{tabular}{ccccccc}
\hline \multirow{2}{*}{ Strain } & \multicolumn{7}{c}{ Parameter } \\
\cline { 2 - 7 } & $\boldsymbol{R}_{\mathbf{0}}$ & $\boldsymbol{r}$ & $\lambda$ & GRR & $\boldsymbol{T}(\mathbf{d})$ & Rate of relative fitness $^{\mathbf{1}}$ \\
\hline SS & $74.80 \pm 5.94 \mathrm{a}$ & $0.23 \pm 0.01 \mathrm{a}$ & $1.26 \pm 0.01 \mathrm{a}$ & $105.25 \pm 7.36 \mathrm{a}$ & $18.59 \pm 0.15 \mathrm{c}$ & 1.00 \\
Sub-1 & $30.15 \pm 3.67 \mathrm{c}$ & $0.18 \pm 0.01 \mathrm{~d}$ & $1.18 \pm 0.01 \mathrm{~d}$ & $75.17 \pm 7.49 \mathrm{~b}$ & $20.34 \pm 0.13 \mathrm{a}$ & 0.40 \\
Sub-6 & $43.40 \pm 4.91 \mathrm{~b}$ & $0.19 \pm 0.01 \mathrm{c}$ & $1.21 \pm 0.01 \mathrm{c}$ & $87.80 \pm 8.52 \mathrm{ab}$ & $19.55 \pm 0.13 \mathrm{~b}$ & 0.58 \\
Sub-12 & $49.08 \pm 5.47 \mathrm{~b}$ & $0.20 \pm 0.01 \mathrm{c}$ & $1.22 \pm 0.01 \mathrm{c}$ & $94.95 \pm 9.17 \mathrm{ab}$ & $19.30 \pm 0.09 \mathrm{~b}$ & 0.66 \\
SubR-12 & $53.41 \pm 5.09 \mathrm{~b}$ & $0.21 \pm 0.01 \mathrm{~b}$ & $1.24 \pm 0.01 \mathrm{~b}$ & $83.68 \pm 7.10 \mathrm{~b}$ & $18.59 \pm 0.14 \mathrm{c}$ & 0.71 \\
\hline
\end{tabular}

Means marked with different letters within the same column are significantly different $(p<0.05$;

Tukey-Kramer test); ${ }^{1} R_{0}$ of selected strain/ $R_{0}$ of SS strain.

\subsection{Abnormalities Caused by Cantharidin Treatment}

Malformation and abnormalities of adults were noticed in the cantharidin treatment (Figure 3) and this progressively became more apparent as the number of sublethal selection cycles increased (Figure 4).

\section{Discussion}

In this study, we found cantharidin maintained a high toxicity in the selected strain (Sub). The susceptibility of the third instar larvae of $P$. xylostella to cantharidin decreased slightly as the number of sublethal selection cycles increased. Similar results were found in P. xylostella treated with sublethal concentration of spinosad [22]. However, such a reduction can be mostly recovered in less than two generations when the selection pressure is eliminated. The SubR strain maintained a stable and high susceptibility similar to the SS strain throughout the following generations.

The selection experiment in our study showed that frequent application of low-dose cantharidin may change the sublethal effects of cantharidin on P. xylostella with time. In general, the sublethal effects on Sub-1 were significant, and these effects were mainly expressed in the differing biological characteristics and demographic parameters when compared with the SS strain. These negative effects still existed in the Sub strain after being selected for 12 generations, although most biological characteristics recovered gradually. 

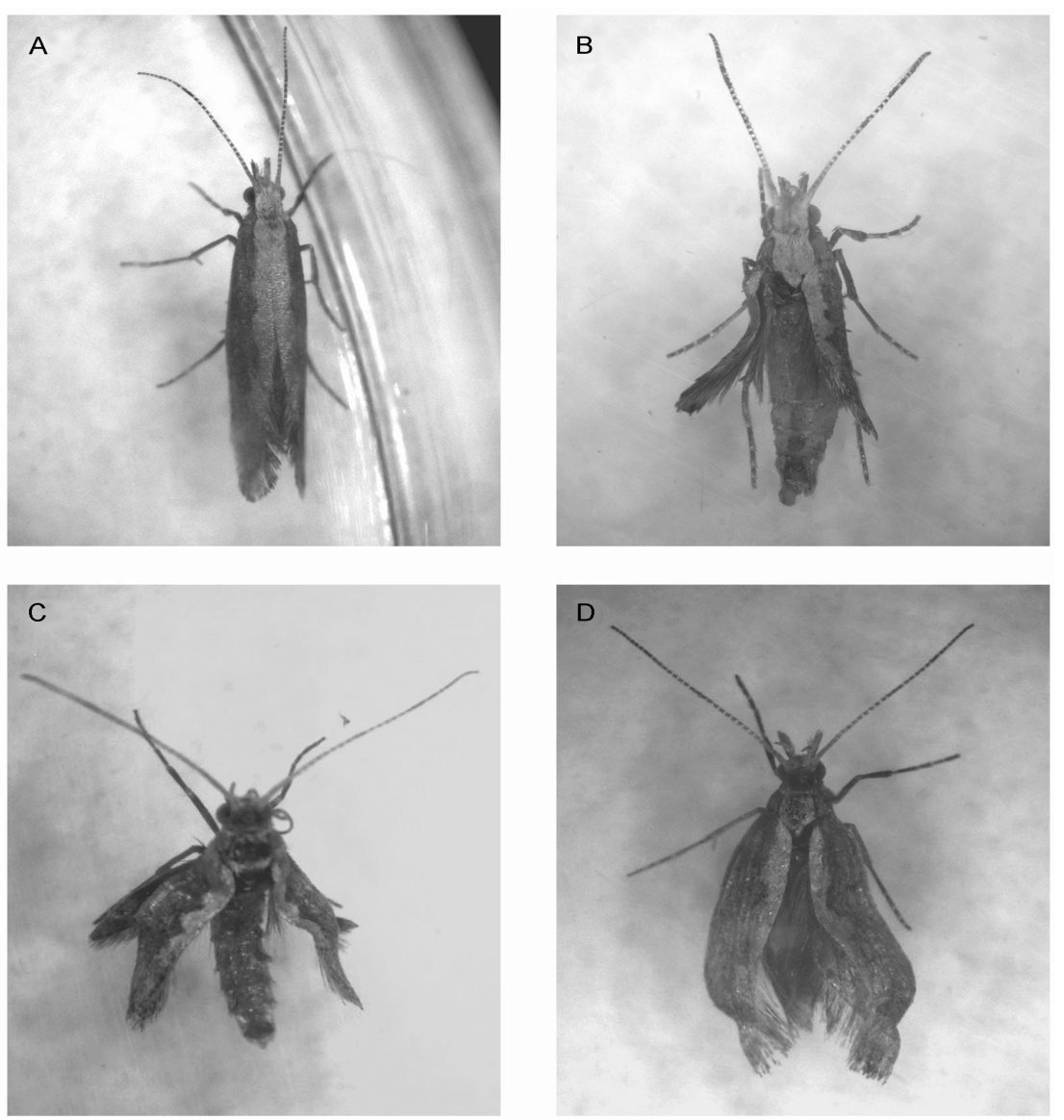

Figure 3. Various morphological abnormalities in treated Plutella xylostella with sublethal concentration of cantharidin. (A) normal adult; (B) miniature crippled wings in adult; $(\mathbf{C}, \mathbf{D})$ twisted wings in adults.

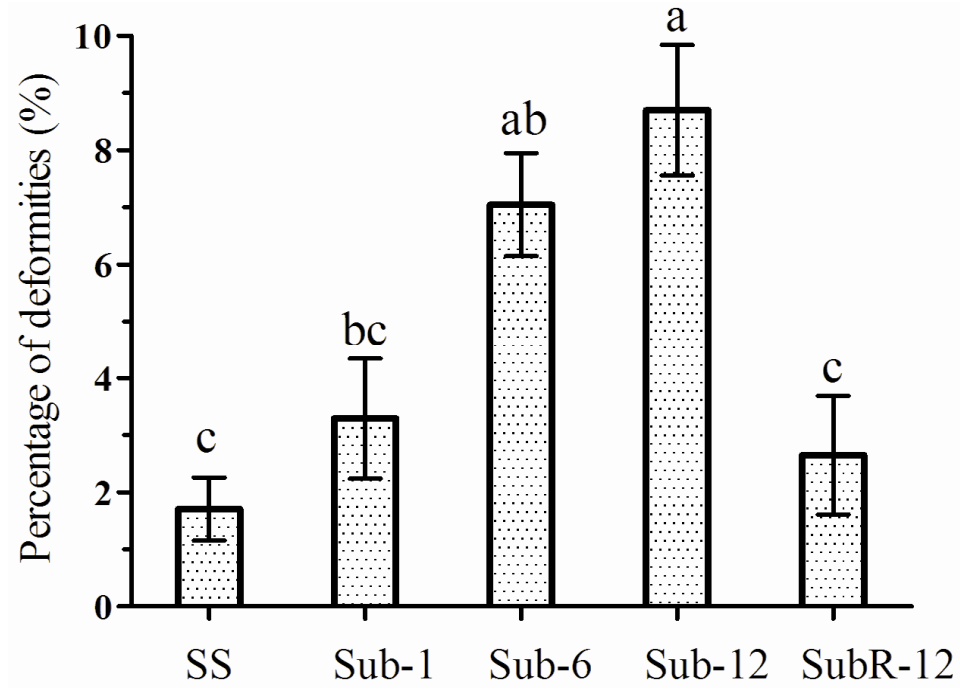

Figure 4. Percentage of abnormalities in different Plutella xylostella strains. The results are presented as means $( \pm \mathrm{SE})$. Histograms bearing different letters are significantly different at the $p<0.05$. (Tukey's HSD test). 
The egg incubation period was not significantly affected whereas larval and pupal development was decelerated strongly in Sub-1 and Sub-6 cohorts. Our finding is consistent with previous reports of short-term exposure [17-19]. A similar negative effect in larval and pupal development of $P$. xylostella was also observed after being exposed to sublethal concentrations of hexaflumuron [27]. A further harmful effect of cantharidin was observed on adult performance of $P$. xylostella. In the current study, fecundity was significantly reduced in Sub-1. Similar phenomena were also found in P. xylostella treated with azadirachtin and neemarin [28,29]. Such reduction on fecundity by sublethal concentrations of insecticides may contribute to depressing future population growth [30]. Previous research suggested that the reduction in fecundity might be related to physiological and morphological changes in females of different orders [31]. Therefore, a sublethal concentration of cantharidin could significantly influence the reproductive capacity of females. However, these effects gradually faded in latter generations. In the present study, the fecundity of the Sub-12 was slightly higher than that of the SS strain, but no mathematically significant difference was observed. Similar result concerning changes in fecundity of Chironomus riparius Meigen exposed to $159.6 \mathrm{nM} \mathrm{Cd}$ was observed, wherein the fecundity gradually recovered after a sharp decrease in the first three generations [32]. One possible reason for this finding may be that the surviving females allocate a specific amount of nutrition across more eggs to maintain high reproductive rates and ensure population stability in the future [33].

P. xylostella eggs became smaller in the first generation under $\mathrm{LC}_{25}$ selection and this smaller size was maintained thereafter. This indicated that selection under cantharidin favors a diminished egg size in P. xylostella. The smaller egg size in the Sub strain may be a parallel phenomenon caused by selection at sublethal doses of cantharidin. A similar phenomenon was found in P. xylostella that treated with fenvalerate [34]. Moreover, in the Sub strain, the survival rate of larvae hatched from the smaller eggs was lower than that from normal-sized eggs in the SS strain. Yin et al. also reported that the survival females laid smaller eggs after the third instar larvae were treated with the $\mathrm{LC}_{25}$ concentration of spinosad, and the smaller eggs showed a lower hatchability [22]. This may be due to the fact that the embryo failed to complete the development phase or perforated the surrounding vitelline membrane [35]. In general, a smaller insect egg results in lower viability in the subsequent developmental stages [36-38]. Females of the SubR strain also laid fewer and smaller eggs, even though they were not exposed to the cantharidin for 12 generations. This suggests that smaller-sized eggs may be a genetic trait and selection by cantharidin produces a diminished egg size in P. xylostella [34]. These results suggest that the SubR strain also has a lower fitness level in the absence of selection pressure.

The demographic toxicological analysis that incorporates life table parameters has been recommended for evaluating the effect of an insecticide $[39,40]$. In this study, the analyses of estimated demographic parameters in the Sub strain provided insights into the adverse effects of sublethal cantharidin on $P$. xylostella on a generational scale. $R_{0}, r$ and $\lambda$ significantly decreased during the first generation (Sub-1). These all imply an adverse impact on population growth in P. xylostella. In addition, $T$ tended to be longer, and the extended $T$ may cause a remarkable deceleration of life cycle and fewer generations per year. By reference to previous studies, insects treated with the sublethal concentration of the same insecticide might exhibit differential effects along with the time of exposure. Vogt et al. reported that the population growth rate was remarkably decreased in the first generation when C. riparius were exposed to low concentrations of tributyltin but for generations seven to ten, the population growth rate tended to recover [21]; Yin et al. demonstrated that the population growth rate 
was significantly decreased in P. xylostella in the first generation against a $\mathrm{LC}_{25}$ dose of spinosad; after five to ten generations, however, the population growth rate gradually recovered [22]. Similar results concerning changes in demographic parameters were observed in our multigenerational experiment. For the following generations in Sub strain, all above-mentioned parameters and the population relative fitness recovered gradually. This may be due to the development of physiological adaptation of P. xylostella to sublethal concentration of cantharidin [41]. Although these effects of demographic parameters can be recovered when the selection pressure is removed, the SubR strain still maintained a disadvantage in relative fitness (0.71) even after 12 generations. Decreased demographic parameters and fitness indicated a profound reduction in the forthcoming generation, thus maintaining the pest population below a level of economic loss, this is of practical importance [42].

Additionally, twisted and crippled wings were observed among both males and females. This could cause flight disability and mating disruption. Similar symptoms were found in previous studies in our lab for H. armigera and the decreased activity of alkaline phosphatase (ALP) was considered as a factor causing these deformities [18]. An earlier study demonstrated that the activity of ALP was significantly lower after cantharidin treatment, and this decrease became more noticeable with the extension of exposure time [6]. We also observed that the changes in morphological traits became more obvious as the number of sublethal selection cycles increased. This showed that cantharidin may take effect over a long period, accumulating damaging sublethal effects later in life.

\section{Materials and Methods}

\subsection{Insects and Chemicals}

An insecticide-susceptible strain of P. xylostella was maintained in our laboratory (Key Lab of Plant Protection Resources \& Pest Management of Ministry of Education, Northwest A\&F University, Yangling, China) for over five years without exposure to any insecticide. Larvae were reared on vermiculite-cultured pakchoi (Brassica chinensis L.) seedlings at $25 \pm 2{ }^{\circ} \mathrm{C}, 50 \% \pm 5 \%$ relative humidity $(\mathrm{RH})$ with a photoperiod of 16:8 (L:D) h. Adults were provided with $10 \%$ honey solution as nutrient.

Cantharidin used in this study was extracted from Mylabris phalerata Pallas (Chinese blister beetle) (purity $>98 \%$ ) and stored in our lab.

\subsection{Bioassay}

A leaf-spray bioassay method [43] was used to determine the susceptibility of $P$. xylostella to cantharidin. Compounds were dissolved in a component solvent (water:acetone $=19: 1, v / v$ ) containing 0.5\% Tween-80 (Shanghai Jingchun Reagent Co., Shanghai, China) as emulsifier. On the basis of preliminary assays, at least seven serial dilutions ranging from 0.625 to $60 \mathrm{mg} \cdot \mathrm{L}^{-1}$, were used. Bioactivity against the healthy third instar larvae was tested using treated cotyledons of pakchoi seedlings [44]. The third instar larvae were identified by head capsule width [45,46]. Cotyledons, were sprayed evenly by test solutions for about $5 \mathrm{~s}$ using a chromatographic sprayer [43], air dried for about $25 \mathrm{~min}$ at room temperature and transferred to Petri dishes $(9 \mathrm{~cm}$ diameter $)$ lined with a slightly moistened filter paper. Four replicates of ten larvae were used per concentration. Treatment with solvent was carried out as the control. Larvae were starved for $4 \mathrm{~h}$ and then released in Petri dishes with ten 
treated cotyledons. Subsequently, larvae were kept in a growth cabinet (ZPQ-280, Dongtuo Instrument Co. LTD, Harbin, China) under the standard conditions mentioned above, and mortality was recorded after $48 \mathrm{~h}$.

\subsection{Selection Experiments}

Based on the bioassay results, $48 \mathrm{~h} \mathrm{LC}_{25}$ concentration was used for the subsequent experiments. The Sub strain (selected by $\mathrm{LC}_{25}$ cantharidin for every generation) was consecutively selected by using the leaf-spray method. Strain Sub-n represents the strain selected for $n$ generations consecutively. The third-instar larvae of each generation were fed with pakchoi cotyledons and treated with $\mathrm{LC}_{25}$ equivalent cantharidin for $48 \mathrm{~h}$ as described above. The larvae fed on the solvent treated cotyledons acted as the SS strain. The SS strain was reared in the laboratory in parallel with the Sub strain. The progeny of the selection-surviving insects were split into two groups: one for the next generation selection, and the other for toxicity testing. The revertant strain (SubR) was derived from a substrain of Sub-12 without exposure to cantharidin or any other insecticides. SubR-n represents the revertant strain without exposure to cantharidin or any other chemicals and insecticides for $\mathrm{n}$ generation(s). The Sub, SubR and SS strains were tested for their susceptibility to cantharidin (the concentration that kills, $\mathrm{LC}_{50}$ ) in each generation.

\subsection{Biological Characteristics of Plutella xylostella in Different Strains}

Demographic observations were conducted for the SS, Sub-1, Sub-6, Sub-12 and SubR-12 cohorts. For each colony, six replicates of fifty newly laid eggs were used. Each egg was placed in a Petri dish (9 $\mathrm{cm}$ diameter) and hatched under the same insect rearing conditions as described above. The hatched larvae were supplied with fresh pakchoi cotyledons that were changed when necessary. The egg incubation, larval, and pupal developmental periods and mortality were recorded every day until all larvae had either died or developed into adults. Following the emergence of adults, the abnormal ones were photographed and the percentage of abnormalities in adults was recorded. Moths that emerged on the same day were paired and allowed to lay eggs until the death of all individuals. The longevity of adults and the duration of pre-oviposition, oviposition and post-oviposition were recorded. In addition, the total number of eggs laid per female and the rates of hatching were recorded for the calculation of fecundity and fertility.

\subsection{Egg Size}

The sizes of 10 eggs laid by females on the first oviposition day from each replicate were measured using a stereo-microscope with an ocular micrometer (SMZ-1500, Nikon Co., Tokyo, Japan). The major axis $(a)$ and minor axis $(b)$ of the egg were measured, and the volume $(V)$ was calculated using the formula $V=\pi a b^{2} / 12$, based on the assumption that the shape was half ellipsoid [47].

\subsection{Age-Stage, Two-Sex Life Table Analysis}

Life-history raw data from different strains were analyzed according to the age-stage, two-sex life table theory $[48,49]$. The age-stage specific survival rate $\left(s_{x j}\right)$ (where $x$ is the age and $j$ is the stage), the age-specific survival rate $\left(l_{x}\right)$ and age-specific fecundity $\left(m_{x}\right)$ were calculated. The $l_{x}$ and $m_{x}$ are estimated as: 


$$
l_{x}=\sum_{j=1}^{k} s_{x j}
$$

and

$$
m_{x}=\frac{\sum_{j=1}^{k} s_{x j} f_{x j}}{\sum_{j=1}^{k} s_{x j}}
$$

The intrinsic rate of increase $(r)$ is calculated iteratively from the Euler-Lotka equation with age indexing from 0 [50]:

$$
\sum_{x=0}^{\infty} e^{-r(x+1)} l_{x} m_{x}=1
$$

The relationship between $R_{0}$ and mean female fecundity, $F$, is defined as follows:

$$
R_{0}=F\left(N_{f}\right) / N
$$

where $N$ is the total number of individuals used for life table study and $N_{f}$ is the number of female adults [51]. The gross reproduction rate (GRR) is as follows:

$$
\mathrm{GRR}=\sum_{x=0}^{\delta} m_{x}
$$

where $\delta$ is the last age of the cohort. The mean generation time $(T)$ is the length of time that a population needs to increase to $R_{0}$-fold of its size (i.e., $e^{r T}=R_{0}$ or $\lambda^{T}=R_{0}$ ) at the stable age-stage distribution. The $T$ is calculated as:

$$
T=\frac{\ln R_{0}}{r}
$$

A computer program, TWOSEX-MSChart [51] was used for data analysis in Visual BASIC for the Windows operating system.

\subsection{Data Analysis}

Bioassay data were collected and analyzed by standard probit analysis, using Abbott's correction [52] for control mortality. The $\mathrm{LC}_{25}$ and $\mathrm{LC}_{50}$ values were estimated from dosage-mortality regression by using the statistical program POLO-PC (LeOra Software Inc., Berkeley, CA, USA, 1987). The lethal concentration (LC) values for different strains were considered significantly different if their $95 \%$ confidence limits (CL) did not overlap. The sublethal effects of cantharidin on P. xylostella biological characteristics were analyzed by one-way ANOVA and Tukey's HSD test $(p<0.05)$ using SPSS 12.0 (IBM Inc., Chicago, IL, USA, 2003). All data were checked for normality using a non-parametric Kolmogorov-Smirnov test $(p<0.05)$ and the fecundity data were $\log (x+1)$ transformed, as necessary, but untransformed means were also presented. The means, variances and standard errors of the demographic parameters were estimated with the bootstrap procedure $(B=10,000)[53,54]$. Mean values of these data were compared using the Tukey-Kramer procedure to admit significant differences at $p<0.05$. The results of $s_{x j}, l_{x}, m_{x}$ and the percentage of deformities were plotted using GraphPad Prism 5 (GraphPad Software Inc., San Diego, CA, USA, 2007). 


\section{Conclusions}

In summary, the consistency of high-level susceptibility of $P$. xylostella to cantharidin was observed through frequent application of cantharidin. Low-dose cantharidin exhibited severe negative effects on population growth throughout the Sub strain and even in the SubR-12, a revertant strain without selection by cantharidin for 12 consecutive generations. Taken together these results indicate that cantharidin serving as an effective alternative to conventional pesticides could be incorporated into integrated pest management program targeting P. xylostella.

\section{Acknowledgments}

We sincerely appreciate Hsin Chi (National Chung Hsing University, Taiwan) for generously providing his software "Life table, two-sex life table analysis" to us. We are grateful to John Richard Schrock (Emporia State University, Emporia, KS, USA) for revising the manuscript. We thank postgraduate Wang Yao for her help during the experiment. This research is supported by the Special Fund for the Public Interest (Agriculture) (200903052) by The Ministry of Science and Technology and The Ministry of Agriculture of China and the "13115" Sci-Tech Innovation Project of Shaanxi Province (2007ZDKG-14).

\section{Author Contributions}

Zhengyu Huang and Yalin Zhang conceived and designed the experiments. Zhengyu Huang performed the experiments. Zhengyu Huang analyzed the data and results. Yalin Zhang contributed reagents, materials, and analysis tools. Zhengyu Huang and Yalin Zhang wrote the paper.

\section{Conflicts of Interest}

The authors declare no conflict of interest.

\section{References}

1. Furlong, M.J.; Wright, D.J.; Dosdall, L.M. Diamondback moth ecology and management: Problems, progress, and prospects. Annu. Rev. Entomol. 2013, 58, 517-541.

2. Wei, H.; Wang, J.; Li, H.S.; Dai, H.G.; Gu, X.J. Sublethal effects of fenvalerate on the development, fecundity, and juvenile hormone esterase activity of diamondback moth, Plutella xylostella (L.). Agric. Sci. China 2010, 9, 1612-1622.

3. Guo, L.; Liang, P.; Zhou, X.; Gao, X. Novel mutations and mutation combinations of ryanodine receptor in a chlorantraniliprole resistant population of Plutella xylostella (L.). Sci. Rep. 2014, 4, 1-7.

4. Talekar, N.S.; Shelton, A.M. Biology, ecology, and management of the diamondback moth. Annu. Rev. Entomol. 1993, 38, 275-301.

5. Zhang, Y.L.; Zhou, Y.; Zhang, Z.Y. Effect of cantharidin on the midgut of oriental armyworm (Mythimnaseparata) and diamondback moth (Plutellaxylostella). Acta Entomol. Sin. 2003, 46, 272-276.

6. Ma, Y.; Liu, R.R.; Ma, Z.Q.; Zhang, Y.L. Effects of cantharidin on four metabolizing enzymes and PPO in Mythimna separata (Walker) (Lepidoptera: Noctuidae). Acta Entomol. Sin. 2010, 53, 870-875. 
7. Khan, R.A.; Rashid, M.; Wang, D.; Zhang, Y.L. Toxicology and biochemical basis of cantharidin effects on Helicoverpa armigera (Hub.) (Lepidoptera: Noctuidae). Pak. J. Zool. 2013, 45, 769-777.

8. Wu, Z.W.; Yang, X.Q.; Zhang, Y.L. The Toxicology and Biochemical Characterization of Cantharidin on Cydia pomonella. J. Econ. Entomol. 2015, doi:10.1093/jee/tou031.

9. Stark, J.D.; Banks, J.E. Population-level effects of pesticides and other toxicants on arthropods. Annu. Rev. Entomol. 2003, 48, 505-519.

10. Stark, J.D.; Rangus, T.M. Lethal and sublethal effects of the neem insecticide formulation, 'Margosan-O', on the pea aphid. Pestic. Sci. 1994, 41, 155-160.

11. Mahmoudvand, M.; Abbasipour, H.; Garjan, A.S.; Bandani, A.R. Sublethal effects of indoxacarb on the diamondback moth, P. xylostella (L.) (Lepidoptera,Yponomeutidae). Appl. Entomol. Zool. 2011, 46, 75-80.

12. Boina, D.R.; Onagbola, E.O.; Salyani, M.; Stelinski, L.L. Antifeedant and sublethal effects of imidacloprid on Asian citrus psyllid, Diaphorina citri. Pest Manag. Sci. 2009, 65, 870-877.

13. Wang, D.X.; Qiu, H.; Ren, X.X.; Zhang, W.Z.; Wang, K.Y. Sublethal effects of spinosad on survival, growth and reproduction of Helicoverpa armigera (Lepidoptera: Noctuidae). Pest Manag. Sci. 2009, 65, 223-227.

14. Tan, Y.; Biondi, A.; Desneux, N.; Gao, X.W. Assessment of physiological sublethal effects of imidacloprid on the mirid bug Apolygus lucorum (Meyer-Dür). Ecotoxicology 2012, 21, 1989-1997.

15. Forbes, V.E.; Calow, P. Is the per capita rate of increase a good measure of population-level effects in ecotoxicology? Environ. Toxicol. Chem. 1999, 18, 1544-1556.

16. Kerns, D.L.; Stewart, S.D. Sublethal effects of insecticides on the intrinsic rate of increase of cotton aphid. Entomol. Exp. Appl. 2000, 94, 41-49.

17. Rashid, M.; Khan, R.A.; Zhang, Y.L. Physiological and population responses of armyworm Mythimna separata (Lepidoptera: Noctuidae) to a sublethal dose of Cantharidin-AC. J. Econ. Entomol. 2013, 106, 2177-2182.

18. Khan, R.A.; Rashid, M.; Wang, D. Zhang, Y.L. Lethal and sublethal effects of cantharidin on the life history traits and population parameters of Helicoverpa armigera (Hübner) (Lepidoptera:Noctuidae). Pest Manag. Sci. 2014, 70, 39-45.

19. Huang, Z.Y.; Wang, Y.; Zhang Y.L. Lethal and Sublethal Effects of Cantharidin on Development and Reproduction of Plutella xylostella (Lepidoptera: Plutellidae). J. Econ. Entomol. 2015, doi:10.1093/jee/tov057.

20. Young, H.P.; Bailey, W.D.; Roe, R.M. Spinosad selection of a laboratory strain of the tobacco budworm, Heliothis virescens (Lepidoptera: Noctuidae), and characterization of resistance. Crop Prot. 2003, 22, 265-273.

21. Vogt, C.; Nowak, C.; Diogo, J.B.; Oetken, M.; Schwenk, K.; Oehlmann, J. Multi-generation studies with Chironomus riparius - Effects of low tributyltin concentrations on life history parameters and genetic diversity. Chemosphere 2007, 67, 2192-2200.

22. Yin, X.H.; Wu, Q.J.; Li, X.F.; Zhang, Y.J.; Xu, B.Y. Demographic changes in multigeneration Plutella xylostella (Lepidoptera: Plutellidae) after exposure to sublethal concentrations of spinosad. J. Econ. Entomol. 2009, 102, 357-365. 
23. Tang, B.; Sun, J.; Zhou, X.; Gao, X.; Liang, P. The stability and biochemical basis of fufenozide resistance in a laboratory-selected strain of Plutella xylostella. Pestic. Biochem. Phys. 2011, 101, $80-85$.

24. Nemoto, H. Factors inducing resurgence in the diamondback moth after application of methomyl. In Diamondback Moth Management, Proceedings of First International Workshop, Tainan, Taiwan, 11-15 March 1985; Talekar, N.S., Griggs, T.D., Eds.; Asian Vegetable Research and Development Ceter: Shanhua, Taiwan, 1986; pp. 387-394.

25. Nemoto, H. Mechanism of resurgence of the diamondback moth, Plutella xylostella (L.) (Lepidoptera: Yponomeutidae). Jpn. Agr. Res. Q. 1993, 27, 27-32.

26. Sota, N.; Motoyama, N.; Fujisaki, K.; Nakasuji, F. Possible amplification of insecticide hormoligosis from resistance in the diamondback moth, Plutella xylostella (Lepidoptera: Yponomeutidae). Appl. Entomol. Zool. 1998, 33, 435-440.

27. Mahmoudvand, M.; Abbasipour, H.; Garjan, A.S.; Bandani, A.R. Sublethal effects of hexaflumuron on development and reproduction of the diamondback moth, Plutella xylostella (Lepidoptera: Yponomeutidae). Insect Sci. 2011, 18, 689-696.

28. Pineda, S.; Martinez, A.M.; Figueroa, J.I.; Schneider, M.I.; Estal, P.D.; Vinuela, E.; Gomez, B.; Smagghe, G.; Budia, F. Influence of azadirachtin and methoxyfenozide on life parameters of Spodoptera littoralis (Lepidoptera:Noctuidae). J. Econ. Entomol. 2009, 102, 1490-1496.

29. Ahmad, N.; Ansari, M.S. Effect of neemarin on life table indices of Plutella xylostella (L.). Crop Prot. 2012, 38, 7-14.

30. Sibly, R.M.; Hone, J. Population growth rate and its determinants: An overview. Philos. Trans. $R$. Soc. B 2002, 357, 1153-1170.

31. Sáenz-de-Cabezón, F.J.; Zalom, F.G.; Thompson, P.B. Residual toxicity of acaricides to Galendromus occidentalis and Phytoseiulus persimilis reproductive potential. Biol. Control 2007, 40, 153-159.

32. Postma, J.F.; Davids, C. Tolerance induction and life cycle changes in cadmium-exposed Chironomus riparius (Diptera) during consecutive generations. Ecotox. Environ. Safe. 1995, 30, 195-202.

33. Walthall, W.K.; Stark, J.D. A comparison of acute mortality and population growth rate as endpoints of toxicological effect. Ecotoxicol. Environ. Saf. 1997, 37, 45-52.

34. Chen, X.D.; Nakasuji, F. Diminished egg size in fenvalerate resistant strains of the diamondback moth Plutella xylostella (Lepidoptera: Yponomeutidae). Appl. Entomol. Zool. 2004, 39, 335-341.

35. Wilson, T.G.; Cryan, J.R. Lufenuron, a chitin-synthesis inhibitor, interrupts development of Drosophila melanogaster. J. Exp. Zool. 1997, 278, 37-44.

36. Rossitor, M.C. Maternal effects generate variation in life history: Consequences of egg weight plasticity in the gypsy moth. Funct. Ecol. 1991, 5, 386-393.

37. Brady, M.E. The significance of egg size variation in butterflies in relation to host plant quality. Oikos 1994, 71, 119-129.

38. Fox, C.W. Egg-size manipulations in the seed beetle Stator limbatus: Consequences for progeny growth. Can. J. Zool. 1997, 75, 1465-1473.

39. Bechmann, R.K. Use of life tables and LC50 tests to evaluate chronic and acute toxicity effects of copper on the marine copepod Tisbe furcata (Baird). Environ. Toxicol. Chem. 1994, 13, 1509-1517. 
40. Stark, J.D.; Banks, J.E.; Acheampong, S. Estimating susceptibility of biological control agents to pesticides: Influence of life history strategies and population structure. Biol. Control 2004, 29, 392-398.

41. Hoy, C.W.; Head, G.P.; Hall, F.R. Spatial heterogeneity and insect adaptation to toxins. Annu. Rev. Entomol. 1998, 43, 571-594.

42. Pineda, S.; Schneider, M.I.; Smagghe, G.; Martínez, A.M.; Del Estal, P.; Viñuela, E.; Valle, J.; Budia, F. Lethal and sublethal effects of methoxyfenozide and spinosad on Spodoptera littoralis (Lepidoptera: Noctuidae). J. Econ. Entomol. 2007, 100, 773-780.

43. Wanner, K.W.; Helson, B.V.; Harris, B.J. Laboratory and field evaluation of spinosad against the gypsy moth, Lymantria dispar. Pest Manag. Sci. 2000, 56, 855-860.

44. Sun, W.B.; Liu, Z.Y.; Zhang, Y.L. Cantharidin and its anhydride-modified derivatives: Relation of structure to insecticidal activity. Int. J. Mol. Sci. 2013, 14, 1-16.

45. Sayyed, A.H.; Rizvi, M.R.; Alvi, A.H. Management of diamondback moth, Plutella xylostella (Lepidoptera: Plutellidae): A lesson from South East Asia for sustainable integrated pest management. Pak. J. Biol. Sci. 2002, 5, 234-245.

46. Saeed, R.; Sayyed, A.H.; Shad, S.A.; Zaka, S.M. Effect of different host plants on the fitness of diamondback moth, Plutella xylostella (Lepidoptera: Plutellidae). Crop Prot. 2010, 29, 178-182.

47. Fujiwara, Y.; Takahashi, T.; Yoshioka, T.; Nakasuji, F. Changes in egg size of the diamondback moth Plutella xylostella (Lepidoptera: Yponomeutidae) treated with fenvalerate at sublethal doses and viability of the eggs. Appl. Entomol. Zool (Jpn.) 2002, 37, 103-109.

48. Chi, H.; Liu, H. Two new methods for the study of insect population ecology. Bull. Inst. Zool. Acad. Sin. 1985, 24, 225-240.

49. Chi, H. Life-table analysis incorporating both sexes and variable development rate among individuals. Environ. Entomol. 1988, 17, 26-34.

50. Goodman, D. Optimal life histories, optimal notation, and the value of reproductive value. Am. Nat. 1982, 119, 803-823.

51. Chi, H. TWOSEX-MSChart: A computer program for the age-stage, two-sex life table analysis. Available online: http://140.120.197.173/Ecology/Download/Twosex-MSChart.rar (accessed on 25 November 2014).

52. Abbott, W.S. A method of computing the effectiveness of an insecticide. J. Econ. Entomol. 1925, 18, 265-267.

53. Huang, Y.B.; Chi, H. Life tables of Bactrocera cucurbitae (Diptera: Tephritidae): With an invalidation of the jackknife technique. J. Appl. Entomol. 2013, 137, 327-339.

54. Alinejad, M.; Kheradmand, K.; Fathipour, Y. Sublethal effects of fenazaquin on life table parameters of the predatory mite Amblyseius swirskii (Acari: Phytoseiidae). Exp. Appl. Acarol. 2014, 64, 361-373.

(C) 2015 by the authors; licensee MDPI, Basel, Switzerland. This article is an open access article distributed under the terms and conditions of the Creative Commons Attribution license (http://creativecommons.org/licenses/by/4.0/). 